\title{
NLRP9b: a novel RNA-sensing inflammasome complex
}

\author{
Cell Research (2017) 27:1302-1303. doi:10.1038/cr.2017.93; published online 21 July 2017
}

\begin{abstract}
Inflammasome sensors recognize pathogens and danger signals and assemble an immune signaling complex, which induces the secretion of pro-inflammatory cytokines IL-1 $\beta$ and IL-18, and pyroptosis. A new study published in Nature now describes a new inflammasome sensor NLRP9b in intestinal epithelial cells, which in concert with the RNA sensor DHX9, recognize short dsRNA from Rotavirus.
\end{abstract}

The inflammasome is a complex of cytosolic proteins which aggregate to mediate proteolytic processing of pro-IL-1 $\beta$ and pro-IL-18 and the poreforming protein gasdermin $\mathrm{D}$, leading to pyroptosis that liberates biologically active IL- $1 \beta$ and IL-18 from the cell. Inflammation and cell death triggered by the inflammasome not only contributes to the host defense against infection, but also regulates the development of inflammatory diseases and cancer [1].

Inflammasome sensors either directly interact with a specific pathogenassociated molecular pattern (PAMP) or danger-associated molecular pattern (DAMP), or respond to a physiological aberration triggered by a PAMP or DAMP [2]. Viral infection of myeloid cells generally leads to activation of the AIM2 or NLRP3 inflammasome. The double-stranded DNA (dsDNA) sensor AIM2 recognizes DNA viruses, whereas the general sensor of stress and damage, NLRP3, recognizes many RNA viruses and even some DNA viruses. How inflammasomes operate in cell types other than myeloid cells in response to viral infection is largely unknown. The dsRNA virus Rotavirus is a clinically important enteric virus which causes diarrhea, dehydration and death in young children [3]. The virus has specific tropism and infects epithelial cells of the small intestine. Although Rotavirus was discovered in the 1970s [4], little is known regarding how host cells infected with this virus trigger an innate immune response.

In a recent study published in $\mathrm{Na}$ ture, Zhu and colleagues identified a cytosolic inflammasome sensor for Rotavirus [5]. The authors first established that infection of suckling mice with Rotavirus induced robust activation of caspase-1 in the ileal tissue, indicating a role for inflammasomes in the pathogenesis of Rotavirus infection. The authors then used RNA sequencing and qPCR analysis to profile the gene expression of NLRs and related proteins in the small intestine and found an elevated expression of genes encoding NLRP6, NLRC4, NAIPs and a previously uncharacterized NLR member, NLRP9b. To elucidate the role of this enigmatic NLR, the authors utilized CRISPR-Cas9 technology to generate mouse strains lacking NLRP9b either in all cells or specifically in intestinal epithelial cells. They found that both $\mathrm{Nlrp} 9 b^{-/}$strains were more susceptible to Rotavirus infection compared to wild-type mice, highlighting a crucial role of NLRP9b in controlling Rotavirus infection [5].

To provide evidence that NLRP9b contributed to inflammasome-dependent responses, the authors measured hallmarks of inflammasome activation in intestinal organoids infected with Rotavirus. Similar to organoids lacking key inflammasome components, Nlrp $9 b^{-/}$organoids produced lower levels of IL-18 and were more resistant to cell death compared with wild-type organoids. Interestingly, NLRP6, a highly expressed NLR in intestinal epithelial cells, also contributed to the host defense against Rotavirus infection [5], raising the possibility that NLRP6 and NLRP9b are co-activated and recruited to the same inflammasome complex, similar to the findings that NLRP3 and NLRC4 are co-recruited to the Salmonella-induced inflammasome in macrophages [6]. Although the role of NLRP6 was not investigated further, these findings collectively revealed, for the first time, that a signaling axis defined by NLRP9b-ASC-Caspase-1-Gasdermin D contributed to anti-Rotavirus immunity in intestinal epithelial cells [5].

The authors turned their focus to the molecular mechanisms leading to the assembly of the NLRP9b inflammasome. They found that infection of Rotavirus in HEK293T cells induced an interaction between human NLRP9 and ASC. Human NLRP9 also coprecipitated Rotavirus RNA, exhibiting a preference for short dsRNA in the form of synthetic low-molecular-weight poly(I:C) molecules. However, mouse NLRP9b did not interact with ASC or poly(I:C) in this overexpression setting. The use of a human cell line in these expression studies could have accounted for the observed differences between human NLRP9 and mouse NLRP9b. For example, endogenous human accessory or mediator proteins expressed in HEK293T cells might have facilitated human NLRP9, but not mouse NLRP9b, in binding RNA and initiating assembly of the inflammasome. Indeed, the authors identified the human RNA helicase DHX9 as an interacting partner of 
both human NLRP9 and viral dsRNA. Furthermore, mouse intestinal organoids lacking DHX9 and infected with Rotavirus produced less IL-18 and were more resistant to pyroptosis compared with wild-type organoids, confirming a role for DHX9 in the activation of the NLRP9b inflammasome (Figure 1) [5].

Identification of NLRP9b as a new inflammasome-initiating sensor raises many new and exciting questions. What are the biological functions of NLRP9b in cell types other than enterocytes? Although expressed in oocytes, ovaries and embryos, NLRP9b is not involved in early embryonic development [7]. Does NLRP9b recognize other RNA viruses? Given that DHX9 binds Alu elements of transcribed regions of host genes [8], how does DHX9 distinguish viral dsRNA and host-derived RNA? Previous studies by the Flavell and Fikrig groups have shown that NLRP6 cooperated with the RNA helicase DHX15 in mediating the recognition of viral RNA, contributing to the host defense against the ssRNA virus EMCV [9]. These studies raise the question of whether there is a universal partnership between DHX and NLR family mem-

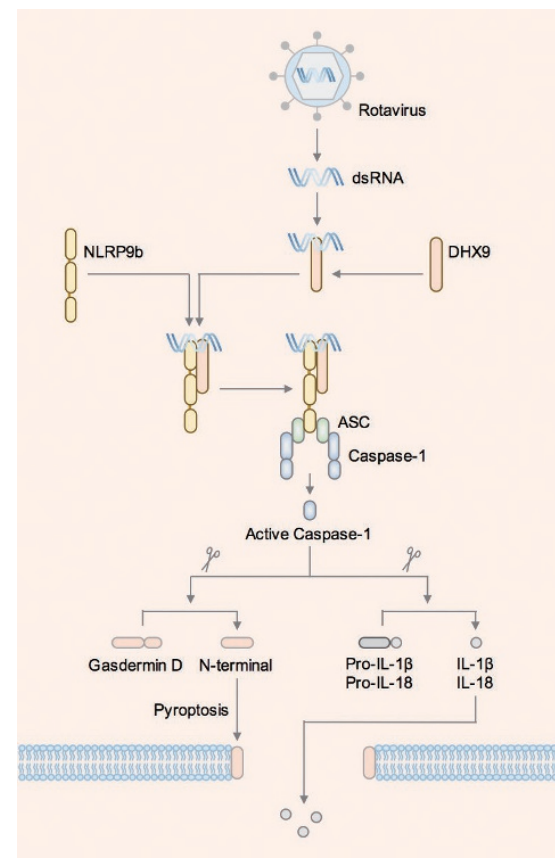

Figure 1 The NLRP9b inflammasome is activated in intestinal epithelial cells during Rotavirus infection. The dsRNA of Rotavirus is recognized by the RNA sensor DHX9, which might bind mouse NLRP9b and activate the inflammasome in intestinal cells.

bers. Addressing these fundamental questions will provide insights into the biological functions of NLRP9b and pattern recognition receptors in health and disease.

\section{Chinh $\mathrm{Ngo}^{1}$, Si Ming Man ${ }^{1}$ \\ ${ }^{I}$ Department of Immunology and Infectious Disease, The John Curtin School of Medical Research, Australian National University, Can- berra, 2601, Australia \\ Correspondence: Si Ming Man \\ Tel: 6161256793 \\ E-mail: siming.man@anu.edu.au}

\section{References}

1 Man SM, Kanneganti TD. Nat Rev Immunol 2016; 16:7-21.

2 Martinon F, Burns K, Tschopp J. Mol cell 2002; 10:417-426.

3 Tate JE, Burton AH, Boschi-Pinto C, et al. Lancet Infect Dis 2012; 12:136-141.

4 Bishop R. J Gastroenterol Hepatol 2009; 24 Suppl 3:S81-S85.

5 Zhu S, Ding S, Wang P, et al. Nature 2017; 546:667-670

6 Man SM, Hopkins LJ, Nugent E, et al. Proc Natl Acad Sci USA 2014; 111:7403-7408.

7 Peng H, Lin X, Liu F, et al. J Reprod Dev 2015; 61:559-564.

8 Aktaş T, Avşar Ilık İ, Maticzka D, et al. Nature 2017; 544:115-119.

9 Wang P, Zhu S, Yang L, et al. Science 2015; 350:826-830. 